${ }^{1}$ Oles Honchar Dnipro National University

${ }^{2}$ National University, Los Angeles, USA

E-mail: lkurdachenko@gmail.com, sasha.pypka@gmail.com, isubboti@nu.edu

\title{
On the influence of ideals and self-idealizing subalgebras on the structure of Leibniz algebras
}

Presented by Corresponding Member of the NAS of Ukraine V.P. Motornyi

The subalgebra A of a Leibniz algebra $L$ is self-idealizing in $L$, if $A=I_{L}(A)$. In this paper we study the structure of Leibniz algebras, whose subalgebras are either ideals or self-idealizing. More precisely, we obtain a description of such Leibniz algebras for the cases where the locally nilpotent radical is Abelian non-cyclic, non-Abelian noncyclic, and cyclic of dimension 2.

Keyword: Leibniz algebra, ideal, idealizer, self-idealizing subalgebra.

Let $L$ be an algebra over a field $F$ with two binary operations + and $[-,-]$. Then $L$ is a left Leibniz algebra, if it satisfies the left Leibniz identity

$$
[[a, b], c]=[a,[b, c]]-[b,[a, c]]
$$

for all $a, b, c \in L$.

Leibniz algebras appeared first in the paper of A. Blokh [1], in which he called them the D-algebras. However, in that time, the work was not in demand, and it had not been properly developed. A real interest to Leibniz algebras appears only after two decades. The term "Leibniz algebra" appeared in the book of J.-L. Loday [2], and article [3]. In [4], J.-L. Loday and T. Pirashvili began a study of the properties of Leibniz algebras. The theory of Leibniz algebras was developed very intensively in many different directions. Some of the results of this theory were presented in book [5]. Note that the Lie algebras are a partial case of Leibniz algebras. Conversely, if $L$ is a Leibniz algebra, in which $[a, a]=0$ for every $a \in L$, then it is a Lie algebra. Thus, the Lie algebras can be characterized as anticommutative Leibniz algebras. In this regard,

Ци ту ванн я: Kurdachenko L.A., Pypka A.A., Subbotin I.Ya. On the influence of ideals and self-idealizing subalgebras on the structure of Leibniz algebras. Допов. Наи. акад. наук Укр. 2021. № 5. С. $12-17$. https://doi.org/10.15407/dopovidi2021.05.012 
a parallel with associative structures, such as, for example, groups and associative rings, comes to mind. There, we can see a significant difference between Abelian and non-Abelian groups, as well as between commutative and non-commutative rings. The differences between Lie algebras and Leibniz algebras became visible, when one considers the first natural types of Leibniz algebras. For example, cyclic Lie algebras have dimension 1, however the structure of cyclic Leibniz algebras is much more sophisticated. The structure of cyclic Leibniz algebras was described in paper [6]. Another example of this sort: Lie algebras, every subalgebra of which is an ideal, is Abelian ones. But, in the case of Leibniz algebras, we have a different more complex case. Leibniz algebras, whose subalgebras are ideals, were described in paper [7]. We face the same situation, when studying other types of Leibniz algebras (see, e.g., [8-11]).

In the current paper, the term "Leibniz algebra" stands for a left Leibniz algebra that is not a Lie algebra.

We start with some definitions and remarks.

Let $L$ be a Leibniz algebra over a field $F$, and let $A$ and $K$ be subalgebras of $L$. The left idealizer of $A$ in $K$ is defined by the following rule:

$$
I_{K}^{\text {left }}(A)=\{x \in K \mid[x, a] \in A \text { for all } a \in A\} .
$$

We note that the left idealizer of $A$ in $K$ is a subalgebra of $K$.

Similarly, the right idealizer of $A$ in $K$ is defined by the following rule:

$$
I_{K}^{\text {right }}(A)=\{x \in K \mid[a, x] \in A \text { for all } a \in A\} .
$$

Unlike a left idealizer, the right idealized of $A$ in $K$ does need not to be a subalgebra. This is shown in the corresponding example from [12, Example 1.7].

The idealizer of $A$ in $K$ is defined by the following rule:

$$
I_{K}(A)=\{x \in K \mid[x, a],[a, x] \in A \text { for all } a \in A\}=I_{K}^{\text {left }}(A) \cap I_{K}^{\text {right }}(A) .
$$

We note that the idealizer of $A$ in $K$ is a subalgebra of $K$.

For an arbitrary subalgebra $A$ of a Leibniz algebra $L$, we have the following ascending series:

$$
A=A_{0} \leqslant A_{1} \leqslant \ldots A_{\alpha} \leqslant A_{\alpha+1} \leqslant \ldots A_{\gamma}
$$

where $A_{1}=I_{L}(A), A_{\alpha+1}=I_{L}\left(A_{\alpha}\right)$ for all ordinals $\alpha$,

$$
A_{\lambda}=\bigcup_{\mu<\lambda} A_{\mu}
$$

for all limit ordinals $\lambda$, and $A_{\gamma}=I_{L}\left(A_{\gamma}\right)$. This series is called the upperidealized series of $A$ in $L$. If $\gamma=1$, then we obtain the following two cases:

$$
A_{1}=I_{L}(A)=L \text { or } A_{1}=I_{L}(A)=A .
$$

The following two types of subalgebras correspond to these two cases: $A$ is an ideal of $L$ or $A$ is self-idealizing in $L$ (i.e., $\left.A=I_{L}(A)\right)$. Therefore, the following natural question appears: what is $a$ 
Leibniz algebra, every subalgebra of which either is an ideal or self-idealizing? This paper shows a description of such Leibniz algebras.

The first type of such algebras is the Leibniz algebras, whose subalgebras are ideals. These algebras were described in [7].

The left (respectively, right) center $\zeta^{\text {left }}(L)$ (respectively, $\zeta^{\text {right }}(L)$ ) of a Leibniz algebra $L$ is defined by the rule:

$$
\zeta^{\text {left }}(L)=\{x \in L \mid[x, y]=0 \text { for each } y \in L\}
$$

(respectively,

$$
\left.\zeta^{\text {right }}(L)=\{x \in L \mid[y, x]=0 \text { for each } y \in L\}\right) .
$$

It is not hard to prove that the left center of $L$ is an ideal, but it is not true for the right center. The right center is a subalgebra of $L$, and, in general, the left and right centers are different. They even may have different dimensions (see, e.g., [13] or [14]).

The center $\zeta(L)$ of $L$ is defined by the rule:

$$
\zeta(L)=\{x \in L \mid[x, y]=[y, x]=0 \text { for each } y \in L\} .
$$

We note then $\zeta(L)$ is an ideal of $L$.

A Leibniz algebra $L$ is called extraspecial, if it satisfies the following condition: the center $\zeta(L)$ is non-trivial and has dimension $1 ; L / \zeta(L)$ is Abelian. An extraspecial algebra $E$ is called a strong extraspecial algebra, if $[x, x] \neq 0$ for each $x \notin \zeta(E)$.

Leibniz algebra $L$, whose subalgebras are ideals, has the following structure: $L=E \oplus Z$, where $Z$ is a subalgebra of the center of $L$, and $E$ is a strong extraspecial algebra [7].

Let $L$ be a Leibniz algebra over a field $F$. Then $L$ includes the greatest locally nilpotent ideal [15, Corollary C1]. This ideal is called the locally nilpotent radical of $L$ and will be denoted by $\operatorname{Ln}(L)$.

Every Leibniz algebra $L$ possesses the following specific ideal. Denote, by Leib $(L)$, the subspace generated by all elements $[a, a], a \in L$. It is possible to prove that $\operatorname{Leib}(L)$ is an ideal of $L$. The ideal Leib $(L)$ is called the Leibniz kernel of $L$.

We note that $L / \operatorname{Leib}(L)$ is a Lie algebra. Conversely, if $H$ is an ideal of $L$ such that $L / H$ is a Lie algebra, then $\operatorname{Leib}(L) \leqslant H$.

The study of Leibniz algebras, whose subalgebras are either ideals or self-idealizing ones, consists of a few stages. At the first stage, we study Leibniz algebras, whose locally nilpotent radical is Abelian and non-cyclic.

Theorem 1. Let L be a Leibniz algebra, whose subalgebras are either ideals or self-idealizing ones. Suppose that the locally nilpotent radical $A$ of $L$ is Abelian. If A is non-cyclic, then the following conditions hold:

(i) $A=\zeta^{\text {left }}(L)$;

(ii) $L=A \oplus W$ where $W$ is a subalgebra of dimension $1, W=F w, W=I_{L}(W)$;

(iii) there exists a non-zero element $\sigma \in F$ such that $[w, a]=\sigma a$ for every $a \in A$.

Conversely, if a Leibniz algebra $L$ satisfies these conditions, then every subalgebra of $L$ is an ideal of $L$ or self-idealizing. 
At the next stage, it is natural to study the case where the locally nilpotent radical is nonAbelian and non-cyclic. Here, we have the following results.

Theorem 2. Let L be a Leibniz algebra over a field $F$, whose subalgebras are either ideals or self-idealizing. Suppose that $L \neq \operatorname{Ln}(L), \operatorname{Ln}(L)$ is a non-cyclic non-Abelian subalgebra. If $[\operatorname{Ln}(L), \operatorname{Ln}(L)] \leqslant \zeta(L)$, then $\operatorname{char}(F)=2$, and the following conditions hold:

(i) $K=\operatorname{Ln}(L)$ is strong extraspecial, moreover $[x, x] \neq 0$ for each $x \notin \operatorname{Leib}(L)$;

(ii) $[K, K]=\zeta(L)=\operatorname{Leib}(L)$;

(iii) $L=K+\langle v\rangle$, where $[v, v]=\eta z$ for some non-zero element $\eta \in F,[v+\zeta(K), x+\zeta(K)]=$ $=x+\zeta(K)$ for each $x \in K \backslash \zeta(K)$.

Example 1. Let $L$ be a Leibniz algebra over a field $F, \operatorname{char}(F)=2$, and let $L$ be generated by the elements $a, b, v$ such that

$$
\begin{aligned}
& {[a, a]=z,[b, b]=\sigma z,[v, v]=\eta z,} \\
& {[z, z]=[z, a]=[a, z]=[z, b]=[b, z]=[z, d]=[d, z]=0,} \\
& {[a, b]=[b, a]=0,[a, v]=a,[v, a]=a+z,} \\
& {[b, v]=b,[v, b]=b+z .}
\end{aligned}
$$

Moreover, the polynomials $X^{2}+\sigma$ and $X^{2}+\eta$ have no root in a field $F$. It is possible to check that $L$ is a Leibniz algebra, whose subalgebras are either ideals or self-idealizing.

Theorem 3. Let $L$ be a Leibniz algebra over a field $F$, whose subalgebras are either ideals or selfidealizing. Suppose that $L \neq \operatorname{Ln}(L)=K$ is non-cyclic and non-Abelian. If $\zeta(L)$ does not include $[K, K]$, then the following conditions hold:

(i) $\operatorname{char}(F) \neq 2$;

(ii) every subalgebra of $K$ is an ideal of $L$;

(iii) $K$ is a strong extraspecial subalgebra;

(iv) $[K, K]=\zeta(K)=\operatorname{Leib}(L)=<z>$ has dimension 1 ;

(v) $L=K+\langle v>$ where $[v+\zeta(K), x+\zeta(K)]=x+\zeta(K)$ for each $x \in K \backslash \zeta(K),[v, z]=2 z$ and $[v, v]=\eta z$ for some $\eta \in F$ ( $\eta$ can be zero).

We say that a field $F$ is 2-closed, if a polynomial $X^{2}+\alpha \in F[X]$ has a root in $F$ for every nonzero element $\alpha \in F$. We note that every finite field $F$ of characteristic 2 is 2 -closed. As a corollary, we obtain that every locally finite field $F$ of characteristic 2 is 2-closed.

Corollary 1. Let $L$ be a Leibniz algebra over a field $F$, whose subalgebras are either ideals or self-idealizing. Suppose that $\operatorname{char}(F)=2, L \neq \operatorname{Ln}(L)$, and $\operatorname{Ln}(L)$ is non-Abelian. If $F$ is 2-closed, then $\operatorname{Ln}(L)$ is cyclic.

Thus, the next natural step is the consideration of the case where the locally nilpotent radical is cyclic. We note that, in this case, it has a dimension 1 or 2 . The following theorem describes the second case.

Theorem 4. Let L be a Leibniz algebra over a field $F$, whose subalgebras are either ideals or selfidealizing. Suppose that $L n(L)$ is a cyclic subalgebra having dimension 2 . Then either $L \neq L n(L)$ or $\operatorname{char}(F)=2$ and $L$ has $a$ basis $\{z, a, v\}$ such that $[z, z]=[z, a]=[a, z]=[z, v]=[v, z]=0,[a, a]=z$, $[v, v]=\eta z,[v, a]=a+\lambda z,[a, v]=a+\mu z, \eta, \lambda, \mu \in F$, and a polynomial $X^{2}+(\mu+\lambda) X+\eta$ has no root in $F$. 
We can show the example of a Leibniz algebra satisfying the conditions of Theorem 4.

Example 2. Let $L$ be a Leibniz algebra over a field $F$, where $\operatorname{char}(F)=2$, and $F$ is not 2-closed. In $F$, choose an element $\eta$ such that a polynomial $X^{2}+\eta$ has no root in $F$. Let $\{z, a, v\}$ be a basis of $L$. Define the operation $[-,-]$ in the following way:

$$
[z, z]=[z, a]=[a, z]=[z, v]=[v, z]=0,[a, a]=z,[v, v]=\eta z,[v, a]=[a, v]=a .
$$

It is possible to check that $L$ is a Leibniz algebra, in which every subalgebra is either an ideal or self-idealizing.

Proposition. Let $L$ be a Leibniz algebra over a field $F$, whose subalgebras are either ideals or self-idealizing. If $\operatorname{Ln}(L)$ has dimension 1, then $L / \operatorname{Ln}(L)$ does not include Abelian subalgebras of dimension 2.

The case where the dimension of the locally nilpotent radical is 1 is reduced to the study of Lie algebras, whose Abelian subalgebras have dimension 1. In this connection, we note that the infinite-dimensional Lie algebras whose proper subalgebras have dimension 1 are some analogs of the Tarski Monster in group theory. The problem of the existence of such Lie algebras is one of the most interesting and difficult unsolved problems in the general theory of Lie algebras.

\section{REFERENCES}

1. Blokh, A. (1965). A generalization of the concept of a Lie algebra. Dokl. Akad. Nauk SSSR, 165, No. 3, pp. 471-473 (in Russian).

2. Loday, J.-L. (1992). Cyclic Homology. Grundlehren der mathematischen Wissenschaften, Vol. 301. Berlin, Heidelberg: Springer. https://doi.org/10.1007/978-3-662-21739-9

3. Loday, J.-L. (1993). Une version non commutative des algèbres de Lie: les algèbres de Leibniz. Enseign. Math., 39, pp. 269-293. https://doi.org/10.5169/seals-60428

4. Loday, J.-L. \& Pirashvili, T. (1993). Universal enveloping algebras of Leibniz algèbras and (co)homology. Math. Ann., 296, No. 1, pp. 139-158. https://doi.org/10.1007/BF01445099

5. Ayupov, S. A., Omirov, B. A. \& Rakhimov, I. S. (2020). Leibniz algebras: structure and classification. Boca Raton: CRC Press, Taylor \& Francis Group.

6. Chupordia, V. A., Kurdachenko, L. A. \& Subbotin, I. Ya. (2017). On some “minimal” Leibniz algebras. J. Algebra Appl., 16, No. 5, 1750082. https://doi.org/10.1142/S0219498817500827

7. Kurdachenko, L. A., Semko, N. N. \& Subbotin, I. Ya. (2017). The Leibniz algebras whose subalgebras are ideals. Open Math., 15, pp. 92-100. https://doi.org/10.1515/math-2017-0010

8. Kurdachenko, L. A., Subbotin, I. Ya. \& Yashchuk, V. S. (2018). Leibniz algebras whose subideals are ideals. J. Algebra Appl., 17, No. 8, 1850151. https://doi.org/10.1142/S0219498818501517

9. Kurdachenko, L. A., Subbotin, I. Ya. \& Semko, N. N. (2018). From groups to Leibniz algebras: common approaches, parallel results. Adv. Group Theory Appl., 5, pp. 1-31. https://doi.org/10.4399/97888255161421

10. Kurdachenko, L. A., Otal, J. \& Subbotin, I. Ya. (2019). On some properties of the upper central series in Leibniz algebras. Comment. Math. Univ. Carolin., 60, No. 2, pp. 161-175.

https://doi.org/10.14712/1213-7243.2019.009

11. Kurdachenko, L. A., Subbotin, I. Ya. \& Yashchuk, V. S. (2020). Some antipodes of ideals in Leibniz algebras. J. Algebra Appl., 19, No. 6, 2050113. https://doi.org/10.1142/S0219498820501133

12. Barnes, D. W. (2011). Some theorems on Leibniz algebras. Comm. Algebra, 39, No. 7, pp. 2463-2472. https://doi.org/10.1080/00927872.2010.489529

13. Kurdachenko, L. A., Otal, J. \& Pypka, A. A. (2016). Relationships between factors of canonical central series of Leibniz algebras. Eur. J. Math., 2, pp. 565-577. https://doi.org/10.1007/s40879-016-0093-5 
14. Kirichenko, V. V., Kurdachenko, L. A., Pypka, A. A. \& Subbotin, I. Ya. (2017). Some aspects of Leibniz algebra theory. Algebra Discrete Math., 24, No. 1, pp. 1-33.

15. Kurdachenko, L. A., Subbotin, I. Ya. \& Semko, N. N. (2018). On the anticommutativity in Leibniz algebras. Algebra Discrete Math., 26, No. 1, pp. 97-109.

Received 20.04.2021

Л.А. Курдаченко ${ }^{1}$, https://orcid.org/0000-0002-6368-7319

О.О. Пипк $a^{1}$, https://orcid.org/0000-0003-0837-5395

I.Я. Субботін ${ }^{2}$, https://orcid.org/0000-0002-6242-3995

${ }^{1}$ Дніпровський національний університет ім. Олеся Гончара

${ }^{2}$ Національний університет, Лос-Анджелес, США

E-mail: lkurdachenko@gmail.com, sasha.pypka@gmail.com, isubboti@nu.edu

ПРО ВПЛИВ ІДЕАЛІВ ТА САМОІДЕАЛІЗОВНИХ

ПІДАЛГЕБР НА БУДОВУ АЛГЕБР ЛЕЙБНІЦА

Підалгебра $A$ алгебри Лейбніца $L €$ самоідеалізовною в $L$, якщо $A=I_{L}(A)$. Вивчено будову алгебр Лейбніца, підалгебри яких або є ідеалами, або самоідеалізовні. Точніше, одержано опис таких алгебр для випадків, коли локально нільпотентний радикал абелевий нециклічний, неабелевий нециклічний, а також циклічний вимірності 2.

Ключові слова: алгебра Лейбніи, ідеал, ідеалізатор, самоідеалізовна підалгебра. 